\title{
Akıllı Telefonda Derin Öğrenme ile Deprem Erken Uyarı Sistemi Tasarımı
}

\author{
Baran Canpolat ${ }^{1}$, Gonca Okay Ahi ${ }^{1 *}$ \\ ${ }^{1}$ Hacettepe Üniversitesi, Mühendislik Fakültesi, Geomatik Mühendisliği Bölümü, Ankara, Türkiye, (ORCID: 0000-0002-8879-6394), b.canpolat@hotmail.com.tr \\ 1*Hacettepe Üniversitesi, Mühendislik Fakültesi, Geomatik Mühendisliği Bölümü, Ankara, Türkiye, (ORCID: 0000-0001-7235-1502), goncaokayahi@hacettepe.edu.tr
}

(İlk Geliş Tarihi 5 Mart 2021 ve Kabul Tarihi 30 Mayıs 2021)

(DOI: 10.31590 /ejosat.891896)

ATIF/REFERENCE: Canpolat, B. \& Okay Ahi, G. (2021). Akıllı Telefonda Derin Öğrenme ile Deprem Erken Uyarı Sistemi Tasarımı. Avrupa Bilim ve Teknoloji Dergisi, (25), 23-27.

\section{Öz}

Ülkemiz gibi deprem kuşağında olan bir coğrafya için deprem araştırmaları ve olası erken uyarı sistemlerine dair olan yeni yaklaşımlar, son zamanlarda meydana gelen depremleri de göz önünde bulunduracak olursak (ör. İzmir, 2020), artan bir önem ve ihtiyaç teşkil etmektedir. Özellikle uyku halinde iken yakalanılan depremler, bilindiği üzere, çok daha vahim sonuçlar doğurmaktadır. Bu çalışmada, mevcut çalışmalardan farklı olarak, ilk tasarımı yapılan deprem erken uyarı sistemi yaklaşımı, uyku halinde iken, içinde bulunan sensörler aracıllğı ile ivmeölçer'e dönüştürülen akıllı telefonlar sayesinde, ReQuakenition ismini verdiğimiz bir telefon uygulaması arayüzü ile acil durumlarda olası bir depremin haber verilmesi amaçlandı. Afet ve Acil Durum Yönetimi Başkanlığı (AFAD) web sayfasından indirilen gerçek deprem verilerinden yararlanarak Uzun kısa süreli belleğe sahip (Long-Short Term Memory: LSTM) tekrarlayan sinir ağı mimarisi (Recurrent Neural Network: RNN) derin öğrenme algoritmaları ile eğitilen verilerden elde edilen sonuçlarda \%82'nin üzerinde duyarlılık gözlemlendi. Elde edilen bu ilk sonuçlar, son derece yaygın olarak kullanılan akıllı telefonların, deprem erken uyarı sistemlerinde kullanılmak üzere, jeodezik ve sismik ağların yanı sıra çok daha yoğun ve homojen bir ivmeölçer ağı gibi çalışabilmesi adına ümit vericidir.

\section{A Preliminary Design of Smartphone-Based Earthquake Early Warning System via Deep Learning}

\begin{abstract}
For an earthquake-prone geography like our country, earthquake studies and new approaches to possible early warning systems are of increasing importance and need, considering especially the recent earthquakes (e.g. Izmir, 2020). As it is known, earthquakes that are occurring especially while in sleep have much more serious consequences. In this study, unlike the current studies, a preliminary earthquake early warning system approach has been designed for the first time to be used while in sleep. It aims to notify a possible earthquake thanks to smartphones that are converted into accelerometers with the sensors inside. A smartphone application interface called ReQuakenition has been also designed for providing a user-friendly tool for emergency cases. By making use of real earthquake data downloaded from the Disaster and Emergency Management Presidency (AFAD) website, $82 \%$ sensitivity was observed in the results obtained from the data trained with the Recurrent Neural Network architecture (RNN) Long-Short Term Memory (LSTM) deep learning algorithms. These initial results are promising for the widely used smartphones to work as a much denser and homogeneous accelerometer network as well as geodetic and seismic networks for use in earthquake early warning systems.
\end{abstract}

Keywords: Smartphones, Accelerometer, Sensor, RNN LSTM, Deep Learning, Earthquake.

*Sorumlu Yazar: goncaokayahi@hacettepe.edu.tr 


\section{Giriş}

Günümüzde dünyada ve Türkiye'de birçok kişi akıllı telefon kullanıcısıdır. Asıl kullanım amaçlarının dışında, bu telefonlar ile hâlihazırda birçok ölçüm yapmak da mümkündür. Bu ölçümleri yapabilmeyi sağlayan içindeki elektronik sensörlerdir. $\mathrm{Bu}$ sensörlere örnek olarak ivmeölçer, GPS alıcısı, jiroskop, barometre, manyetometre verilebilir. Çeşitli bilgileri sağlamak için kullanılan bu sensörler dolaylı olarak biz farkında olmasak da günlük hayatımızda birçok işimizi görmektedir. Bunlar, park etmek (Liu vd., 2012a), fiziksel aktivite tanımlanması (Hassan vd., 2018; Lima vd.,2019; Voicu vd., 2019), insan hareketlerini takip etme (Del Rosario vd., 2015), kalp ritmi takibi gibi tıbbi amaçlı uygulamalar (Majumder ve Deen, 2019) olarak örneklendirilebilir.

Yerbilimlerine yönelik uygulamalara göz atacak olunursa, jiroskop ve ivmeölçer sensörlerinin Kalman filtre kullanılarak birleştirilmesi sonucunda yükseklik tahminin elde edilmesi (Retscher 2019), inersiyal ve magnetik sensörlerin kullanımı ile kapalı alan navigasyonu konularında yapılmış çalışmalar mevcuttur (Manos vd., 2019; Chen vd., 2015; Zhuang vd., 2016; Liu vd., 2012b; Gang ve Pyun, 2019; Ehrlich ve Blankenbach, 2019). Daha fazla bilgi için, akıllı telefonlarda geliştirilen yerbilimleri uygulamalarına dair özet bir çalışma (Lee vd., 2018)'da bulunabilir. Aslında, yerbilimleri uygulamaları denince ilk akla deprem araştırmaları/erken uyarı sistemleri gelmektedir.

Küresel olarak deprem erken uyarı sistemleri ile ilgili geliştirilen yaklaşımlar ve ülkelerin geldiği noktaları anlatan özet bir çalışma (Allen vd., 2009)'da verilir. Bu kaynakta açıklandığı şekilde kavramlara açıklık getirmek adına bu yaklaşımlar şu şekilde özetlenebilir: Öncül tanımlama (Front detection), P dalgası kullanılması, yerinde uyarı (Onsite Warning), Bölgesel uyarı, Jeodezik ağlar. Öncül tanımlama, bir alanda meydana gelen kaynak olarak ifade edeceğimiz güçlü yer sarsıntısı ile uyarı iletilecek kişiler arasında mevcut bulunan sensörler aracılığı ile kaynakta meydana gelen sismik enerjinin uyarısının iletilmesini ifade eder. $\mathrm{Bu}$ yöntem, gelecekteki olası depremlerin konumlarının iyi bilinmesini gerektirir. P dalgası kullanılmasına değinecek olunursa $\mathrm{P}$ dalgasının genliği depremin yaratabileceği olası tahribat hakkında tahmin ve merkezüssü uzaklığı hesabını yapmaya olanak sağlar. Yine elde edilen dalga formundan deprem büyüklüğü (magnitüdü, M) hakkında bilgi sağlanır. Yerinde uyarının temeli bir istasyonda meydana gelen sismik enerjiyi algılamak ve aynı istasyonda tekrar meydana gelecek olan yersarsıntısının uyarısını, P dalgası genliği ve yersarsıntısı arasındaki ilişkinin irdelenmesi sonucu, sağlamaktır (Ör. UrEDAS). Bölgesel uyarı sistemi bir grup sismik ă̆ kullanılarak, bu ağın algıladığı dalga formlarının bir veri-işlem merkezinde algılanmas1, değerlendirilmesi ve raporlanmasından ibarettir. Jeodezik ağlar'a değinecek olunursa bu ağlar, gerçek zamanlı olarak depremlerin algılanması, konumunun ve büyüklüğünün tespit edilmesi konusunda ağ sıklığ i ile doğru orantılı bir şekilde başarılıdır.

Teknolojideki gelişmelere paralel, bu yaklaşımlara ek olarak, yakın zamanda, cep telefonlarının, deprem erken uyarısı için bir araç haline gelmesi söz konusudur. Bu kapsamda, birçok uygulama geliştirildi. Bunlara örnek olarak, Earthquakes Tracker, Earthquake network (https://www.sismo.app) verilebilir. Fakat bu çalışmalar, bir harita üzerinde meydana gelen depremlerin bilgisini edinmek, bir deprem olduğunda bunu işaretleyerek diğer kullanıcılara bilgi vermek amaçlı kullanan bir nevi coğrafi bilgi sistemi olarak iş görmektedir. Ek olarak,
Uluslararası Amerikan Jeoloji derneği (USGS), Kaliforniya Jeoloji araştırmaları derneği, Kaliforniya teknoloji enstitüsü, Kaliforniya acil hizmetler ofisi ve Google gibi birçok kurumun ortaklaşa işbirliği halinde geliştirdiği Shakealert uygulaması örnek verilebilir. Fakat bu uygulama, sismik ve jeodezik ağlardan alınan sinyallerin cep telefonları aracılığı ile cep telefonu kullanıcılarına bir uyarı şeklinde iletilmesini amaçlar ve bu ağların kısıtlı yayılımı sebebiyle belli bir bölge için sınırlıdır. Bunun dışında, Berkeley Üniversitesinde geliştirilen MyShake uygulamasından bahsedilebilir (Kong vd., 2016a; Kong vd., 2016b, Allen vd., 2020). Fakat kullanıcı yorumlarına bakıldığında deprem sinyali ile koşmak, yürümek gibi diğer aktivitelerden ayırt edilse de deprem olmayan sarsıntıları depremden ayırt etmede başarılı olmadığını söylenebilir.

$\mathrm{Bu}$ çalışmada, yukarıda bahsi geçen deprem erken uyarı yaklaşımlarından farklı olarak, akıllı telefonlarda bulunan ivmeölçer sensörü yardımı ile cep telefonunun zamana bağlı ölçü alan bir ivmeölçere dönüştürülmesi amaçlandı. Öncelikle, uyku halinde iken, deprem erken uyarı sistemi olarak tasarlanan bu yaklaşımda, deprem anında algılanan ivme daha önce gerçek deprem verisi kullanılarak derin öğrenme algoritmaları ile deprem bilgisi öğretilmiş sunucuya aktarılacak ve deprem olup olmadığ sunucuda değerlendirildikten sonra kullanıcılara gerekli uyarı aktarılacaktır. Çoklu kullanıcı deneyimi ile aynı lokasyondaki belli sayıdaki kişiden alınan benzer sinyal ile uyarının duyarlılığının artırılması ilerisi için ayrıca öngörüldü. Yukarıda verilen Giriş kısmından sonra ikinci bölümde kullanılan materyal ve yöntem açıklandı. Çalışmanın üçüncü bölümünde araştırma sonuçları ve tartışma sunuldu. En son kısım olan dördüncü bölümde genel sonuçlar verildi.

\section{Materyal ve Yöntem}

$\mathrm{Bu}$ çalışmada, yöntemsel olarak birkaç adım izlendi. Öncelikle cep telefonlarında bulunan 3-bileşenli vektörel ölçüm yapmaya olanak sağlayan ivmeölçer, jiroskop ve bunların dışında GPS sensörlerinin kaydettiği ölçüm verilerinin gerçek zamanlı olarak elde edilebilmesi için Java tabanlı bir yazılım geliştirildi. Sonraki aşamada, Afet ve Acil Durum Müdürlüğü (AFAD) web sayfasındaki veri tabanı kullanılarak, Türkiye'nin çeşitli bölgelerinde gerçekleşmiş, moment büyüklüğü $(\mathrm{Mw}) \geq 4.5$ olan 48 işlenmiş 44 ham veri olacak şekilde toplamda 92 adet ivmeölçer zaman serisi verisi indirildi. AFAD'ın web sayfasından indirilen gerçek deprem ivmeölçer zaman serisi verileri, sonraki aşamada derin öğrenme algoritmalarına depremin öğretilmesi için eğitim ve test seti olarak değerlendirildi. Daha sonra, tüm bunların kullanıcılara olası bir erken uyarı sistemi aracı olarak sunulması için, Java tabanlı, Android işletim sisteminde çalışan ReQuakenition ismini verdiğimiz bir cep telefonu uygulaması geliştirilip ilk tasarımı yapıldı. Akıllı telefonlarına bu uygulamayı yükleyen ve tercihen sürekli ve/veya uyku halinde iken ivmeölçer verisi kaydı alınan kullanıcılardan olası bir deprem kaynaklı algılanan ivme hareketi durumunda bu sinyal çevrimiçi sunucuya gönderilmek üzere tasarland. Mobil platformlar için Google tarafından geliştirilen Firebase adlı sunucuda daha önce derin öğrenme ağları ile eğitilen deprem verisi ve öngörülen çoklu kullanıcı deneyimi sayesinde duyarlılığın artırılması planlanan ve bu şekilde işlenen veri yakını civardaki, ya da derin uyku halindeki kullanıcılara depremin önceden haber verilmesi için tasarlandı. Şekil 1'de bahsi geçen sunucu ile olan veri-alışverişi gösterilmektedir. 


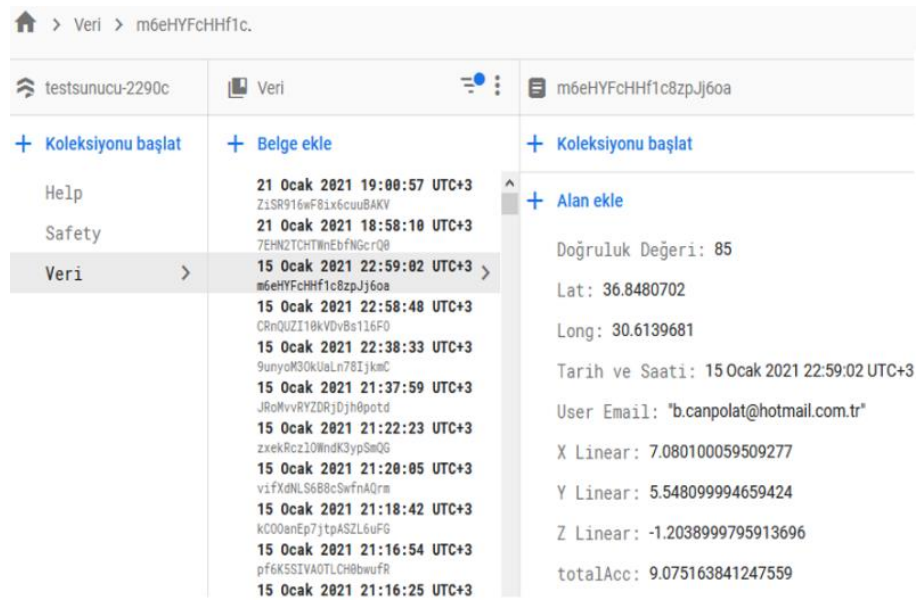

Şekil 1. Firebase'de gerçek zamanlı çevrimiçi veritabanı örnek kaydı.

\subsection{Materyal}

\subsubsection{Sensör Verisi}

Android'in geliştirdiği sensör işletim platformu aracılığı ile cep telefonundan elde edilen zamana bağlı 3-bileşenli doğrusal ivmeölçer verisi $\left(\mathrm{cm} / \mathrm{sn}^{2}=\mathrm{gal}\right)$, ham verinin işlenerek gürültü etkisi azaltılmış ve her eksenden gravite etkisi çıkarılmış halidir (Allen vd., 2009). Ayrıca, elde edilen değerlere, sıcaklık, bias düzeltmesi ve ölçek düzeltmesi uygulanır https://source.android.com/devices/sensors/sensor-types. Şekil 2'de ak1llı telefonlarda kullanılan koordinat sistemi gösterilmektedir.

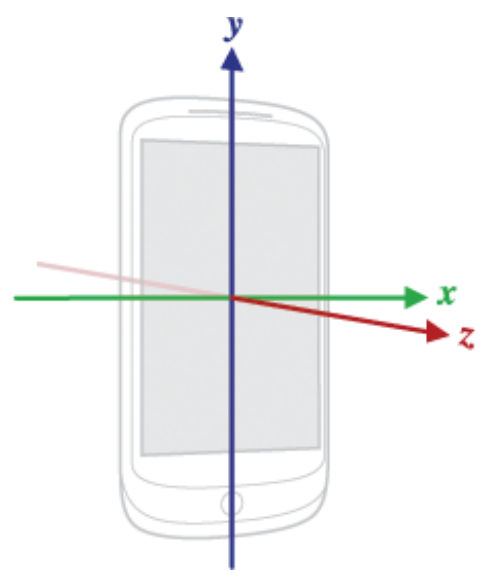

Şekil 2. Akıllı telefonlarda kullanılan üç boyutlu Kartezyen koordinat sistemi.

Bu projede, veri örnekleme aralığg $50 \mathrm{~Hz}$ olarak alındı. Ayrıca ölçüm koşulu olarak kullanılmak üzere, bir gravite sensörü büyüklüğü elde edildi. $\mathrm{Bu}$ cep telefonunda hali hazırda bulunmayan fakat genellikle ivmeölçer, jiroskop gibi sensörlerin Kalman filtresi teknikleri ile sensör füzyonu ile türetilen bir büyüklük olup, üç boyutlu olarak gravite'nin büyüklük ve yön bilgisini sağlamaktadır. Bu veri bize telefonun rotasyon bilgisini aynı zamanda telefon ancak düz bir zemine yerleştirildiği zaman ölçü alınması koşulunun sağlanmasını sağlamaktadır. Bunun dışında acil durumda kullanılmak üzere gerekli olan konum bilgisi GPS alıcıları sayesinde elde edilmektedir.

\subsubsection{Gerçek Deprem Verisi}

Bu çalışmada eğitim ve test veri seti olarak kullanılan veriler AFAD'ın web sitesinden indirildi https://tadas.afad.gov.tr/map. Akıllı telefonların olası ölçüm hassasiyeti (Horvath vd., 2016) göz önüne alındığında, Türkiye'de gerçekleşmiş $\mathrm{Mw} \geq 4.5$ olan verilerin seçilmesine özen gösterildi. Bu büyüklük aynı zamanda hasara sebep olabilecek dikkate alınması gereken büyüklük değeridir. Veri tabanında bu koşulu sağlayan yaklaşık 860 kayıt olmasına rağmen bunların yarısından fazlası denizde olan depremlerdir. Diğerlerinin büyük çoğunluğu düşük ivme değerlerine sahip olması sebebiyle elendi. Ham ve işlenmiş veriler dahil, X (D-B), Y(K-G), Z bileşenleri olacak şekilde model eğitimi için kullanabilinecek toplamda 48 işlenmiş 44 ham toplamda 92 adet veri mevcut bulunmaktadır. İşlenmiş veri olarak ifade edilen veri, ham veriye 2 . dereceden butterworth filtresi uygulanması ile elde edilen veridir ( $f_{\text {low_cut: }} 0.025 \mathrm{~Hz}$, $\mathrm{f}_{\text {high_cut }}: 40 \mathrm{~Hz}$ ). Şekil 3'de kullanılan bazı verilerin genel özellikleri Şekil 4'te ise gerçek bir depreme ait işlenmiş ivmeölçer zaman serisi örneği gösterilmektedir.

\begin{tabular}{|c|c|c|c|}
\hline \multicolumn{2}{|r|}{ Location } & Date & Magnitude (Mw) \\
\hline & Elazığ & 27.12 .2020 & 5.3 \\
\hline & Malatya & 04.08 .2020 & 5.2 \\
\hline & Afyon & 03.02 .2002 & 6.5 \\
\hline Marmara & Denizi(İstanbul) & 26.09 .2019 & 5.8 \\
\hline & Elazığ & 08.03 .2010 & 5.8 \\
\hline & İzmir & 17.10 .2005 & 5.8 \\
\hline & İzmit & 13.09 .1999 & 5.8 \\
\hline & Muğla & 13.04 .2017 & 5.0 \\
\hline & Denizli & 20.03 .2019 & 4.5 \\
\hline & Ankara & 15.03 .2008 & 5.0 \\
\hline
\end{tabular}

Şekil 3. Eğitim ve test verisi için kullanılan gerçek deprem verilerinden bazılarına örnekler.

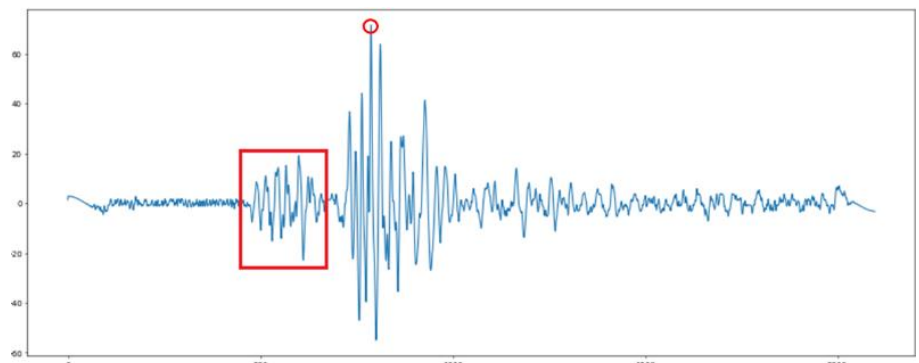

Şekil 4. D-B bileşende işlenmiş örnek bir veri (Ankara 27.12.2007/Mw=5.2). En büyük ivme $\sim 70$ gal'dir (daire sembolü) ve oldukça şiddetli bir dalganın gelmekte olduğunun habercisidir.

Bölüm 2.2'de anlatılacak olan konu ile alakalı Şekil 4'te gözüken kırmızı dikdörtgen ile sınırlı aralık, model eğitim verisi için her deprem verisinden, depremin ivme değerinin artmaya başladığı veya depremin belirgin ilk anından itibaren 80 ölçümlük aralığ 1 ifade etmektedir.

\subsection{Yöntem: Derin Öğrenme (RNN LSTM)}

Cep telefonu sensörlerinden alınan veri kıymetli olmakla birlikte kişinin günlük hayatında gerçekleştirdiği birçok fiziksel aktivite sebebiyle (ör. Yürümek, koşmak, telefonu düşürmek) aslında karmaşıktır. Dolayısıyla, kullanılacak derin öğrenme algoritması ihtiyacımız olan ivmeölçer verisini bu diğer aktivitelerden ayırabilecek nitelikte olmalıdır. $\mathrm{Bu}$ amaçla kullanılan algoritmalar içerisinden, zamana bağlı olarak değişen veri setleri için uygun olan (Lipton vd., 2015), tekrarlayan sinir ağı mimarisinin (RNN: Recurrent Neural Network) özel bir türü 
olan uzun kısa süreli bellek ağları (LSTM: Long-Short Term Memory) modeli seçildi (Sepp Hochreiter ve Juergen Schmidhuber 1997).

RNN sinir ağı geçici hafizaya sahip olması sebebiyle diğer yapay sinir ağlarından farklıdır. Öğretilen bir bilginin hafızadan kullanılabilmesi özelliği sayesinde başka sinir ağı yapılarına kıyasla daha verimli çalışır. Fakat bu hafıza kısa süreli bir belleğe sahiptir. Bu sorunun üstesinden gelebilmek adına RNN'nin özel bir türü olan hem uzun hem kısa süreli belleğe sahip LSTM yapısı geliştirildi. Bu vesile ile sinir ağları yakın ve uzak geçmişe ait bilgileri saklayabilmektedirler. RNN Şekil 5 'de görüldüğü üzere basitçe tekrarlayan bir sinir ağı yapısından oluşur.

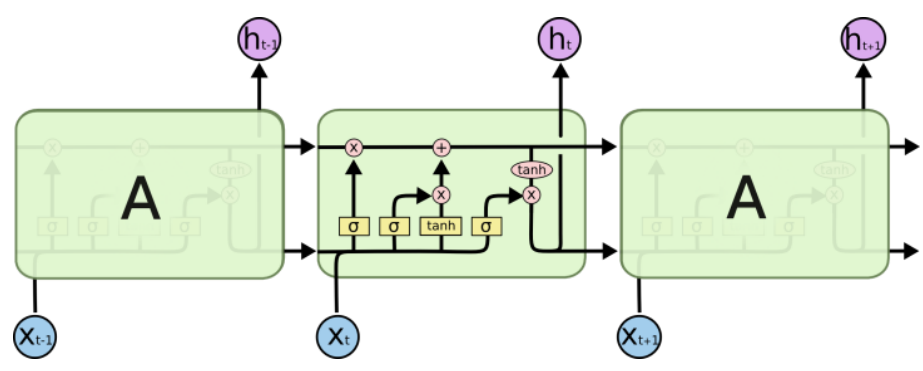

Şekil 5. Uzun kısa süreli belleğe sahip tekrarlayan sinir ağ mimarisi (RNN LSTM). http://colah.github.io/posts/2015-08Understanding-LSTMs/

Burada, A: sinir ağı parçası, $x_{t}$ : girdi veri ve $H_{t}$ çıktı veri olarak adlandırılabilinir. RNN'in özel bir türü olan LSTM'de standart olarak kullanılan tek bir gizli katman yerine 4 adet sinir ağı katmanına rastlanır (şekilde sarı renkli kutular). Bu gizli katmanlar bilgileri hatırlayan kısımdır ve özel bir etkileşime girerek bilgiyi iletir. Bu modelle ilgili detaylı teorik bilgi için Sherstinsky (2020)'ye bakınız. Yapılan bu çalışmada, RNN mimarisinde toplam 1 LSTM katmanı (32 birim) 2 sik (ilki 32 birim diğeri 1 birim) adet katman yapısı kullanıldı. Katmanlar oluşturulduktan sonra optimizasyon yöntemi olarak Relu (rectified linear activation function), hata yöntemi olarak ortalama kare hata (Mean squared error) kullanıldı. Model Tensorflow ve Keras kütüphaneleri kullanılarak tasarlandı Android sistemine olan entegrasyonu ise ücretsiz olarak kullanıcılara sunulan Tensorflow lite versiyonu kullanılarak elde edildi.

\subsection{ReQuakenition uygulaması}

Uygulama yüklendiği zaman kullanıcıdan öncelikle konum, ad soyad, email bilgilerinin girilmesi istenir. Tasarlanan bu uygulama arka planda sürekli olarak çalışmaktadır. Yine de cep telefonun şarjını ve hafızasını optimum seviyede kullanacak şekilde programland. Türetilmiş gravite verisi ile tespit edebileceğimiz üzere, asıl veri cep telefonu düz bir zeminde iken alınacak olmak üzere tasarlandı. Böylece, ilave bir fiziksel aktivite tanımının programlanmasına ihtiyaç kalmadı. Bu ilk örnek tasarımda amaçlanan, gece uyku halinde ani yakalanılan depremlerin önceden haber verilebilmesidir. Geliştirilen mobil uygulamada, sunucuda işlenen ve deprem olduğuna karar verilen gerçek zamanlı sinyal tüm kullanıcılara uyarı olarak iletilecektir. Bu şekilde, deprem merkez üssüne yakın oturan ve etki alanındaki kişiler aynı anda haberdar edilecektir. Bunun dışında uygulama açıldığında AFAD ve/veya Kandilli web sayfası üzerinden sunulan bilgiler aracılığı ile gerçek zamanlı olarak Türkiye'de olan depremlerin konum ve büyüklük bilgilerinin gösterilmesini, olmuş depremin bilgisinin girilmesini, deprem anında şebekeleri meşgul etmemek adına önceden tanımlanmış yakın kişiler listesine "güvendeyim" bilgisinin gönderilmesini öngörmektedir. Deprem olduğu tespit edilen bölgede kayıtlı kullanıcılar tarafından "güvendeyim" bilgisi göndermemiş olanların son konum bilgilerinden hareketle göçük altında kalmış olabileceği öngörülecek ve gerekli arama kurtarma ekiplerine ilgili bilgiler gönderilecek şekilde uygulamanın tasarımı halen geliştirilmektedir. Bu bağlamda ayrıca "yardım" butonu da bulunmaktadır. Şekil 6 geliştirilen uygulamanın ilk tasarımı ve logosu ile ilgili bilgi bulunmaktadır.
A

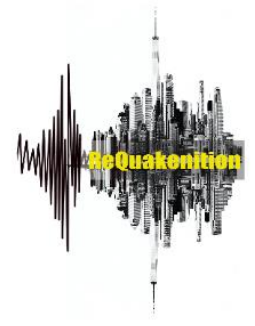

B

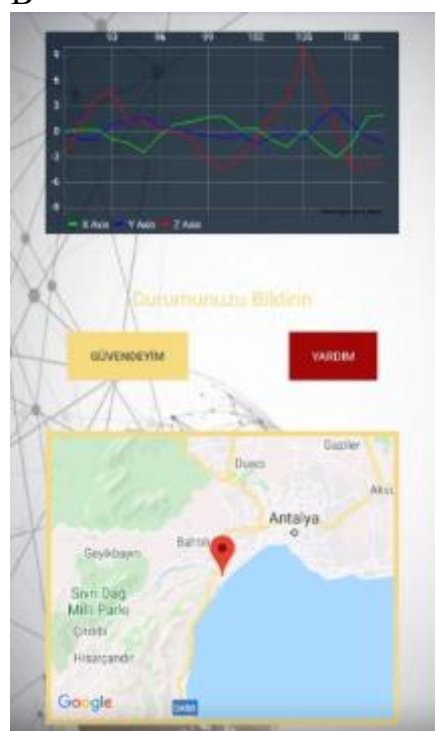

Şekil 6. (A) Deprem erken uyarı için geliştirilen ReQuakenition mobil uygulama logosu ve (B) programın arayüzü.

\section{Araştırma Sonuçları ve Tartışma}

Gerçek deprem verileri ile eğitilen derin öğrenme modelini, yine gerçek ancak modelin eğitimine dahil edilmemiş bir deprem verisi ile test ettiğimizde $\% 96$ gibi bir doğruluk elde edildi. Şekil 7'de elde edilen modelin eğitim doğruluğu grafiği bulunmaktadır.



Şekil 7. Model eğitim doğruluğu grafiği.

Yine aynı şekilde, Python/Random kütüphanesi ile oluşturulan \pm 10 değer aralığına sahip rastgele ondalık sayılar ile elde edilen zaman serisi ile test edilen modelin çıktısı $\% 0$ oldu. $\mathrm{Bu}$ testler göz önüne alındığında ilk aşamada başarılı bir model elde edildiği söylenebilir. 


\section{Sonuç}

$\mathrm{Bu}$ çalışma, deprem erken uyarı sistemleri çalışmalarına teknolojik gelişmelere paralel olarak farklı bir yaklaşımla cep telefonlarının kullanımını dahil etti, bunun üzerinden geliştirilen bir sistem tasarlandı ve mevcut sensörler kullanılarak cep telefonları bir ivmeölçer'e dönüştürüldü. $\mathrm{Bu}$ şekilde günümüzdeki cep telefonu kullanıcı sayısı düşünülecek olunursa, sismik ve jeodezik ağların kısıtlı sayıda olması ve homojen dağılmamasının getirdiği dezavantajların ortadan kaldırılması hedeflendi. RNN LSTM derin öğrenme algoritmaları kullanılarak ögretilen model sayesinde yapılan öncül testlerde tahmin edilen verilerin doğruluğu \%82'nin üzerindedir. Öngörülen çoklu kullanıcı deneyimleri sayesinde bu duyarlılığın daha da arttırılması olasıdır. Yapılan bu çalışma prototip niteliğindedir. Yakın gelecekte bu çalışmanın daha da genişletilmesi adına farklı şiddetteki depremleri simüle etmeye yarayan sarsma tablaları üzerinde farklı sensör duyarlılığındaki akıllı telefonlar kullanılarak, telefonlarda kullanılan sensörlerin farklı deprem büyüklüklerine olan duyarlılığının incelenmesi öngörüldü. Ayrıca, aynı lokasyondaki veriler ya da farklı şiddetteki veriler kullanılarak farklı şekillerde veri girdi setleri çeşitlendirilerek modelin buna duyarlılığının araştırılması da ayrı bir çalışma konusu olarak değerlendirilecektir. Bunlara ek olarak, çalışmanın ileri aşamalarında test için rastgele verilerin yanısıra, hasara sebebiyet vermeyen deprem verilerinin ve depremsiz anlardaki verilerin de test edilmesi faydalı olacaktır. Son olarak, geliştirilen mobil uygulamanın tasarımı ile ilgili kullanıcı ihtiyaçlarını karşılayacak yeni eklentilerin dahil edilmesi planlanmaktadır.

\section{Kaynakça}

Allen R.M., Kong, Q., Martin-Short, R. (2020). The MyShake Platform: A Global Vision for Earthquake Early Warning. Pure Appl Geophys. doi: 10.1007/s00024-019-02337-7

Allen, R. M., Gasparini, P., Kamigaichi, O., \& Bose, M. (2009). The status of earthquake early warning around the world: An introductory overview, Seismological Research Letters, 80(5), 682-693.

Chen, Z., Zou, H., Jiang, H., vd. (2015). Fusion of WiFi, smartphone sensors and landmarks using the kalman filter for indoor localization, Sensors (Switzerland). doi: 10.3390/s150100715

del Rosario, MB., Redmond, SJ., Lovell, NH. (2015). Tracking the evolution of smartphone sensing for monitoring human movement, Sensors (Switzerland).

Gang, H-S., Pyun, J-Y. (2019). A Smartphone Indoor Positioning System Using Hybrid Localization Technology, Energies, 12(19), 3702.

Hassan, MM., Uddin, M.Z., Mohamed, A., Almogren, A. (2018). A robust human activity recognition system using smartphone sensors and deep learning, Futur Gener Comput Syst. doi: 10.1016/j.future.2017.11.029.

Hochreiter, S., \& Schmidhuber, J. (1997). Long short-term memory. Neural computation, 9(8), 1735-1780.

Horvath, Z., Jenak, I., Wu, T., Xuan, C. (2016). Sensitivity of sensors built in Smartphones. In: Harmony Search Algorithm, Springer, 305-313.

Kong, Q., Allen, R. M., Schreier, L., \& Kwon, Y. W. (2016a). MyShake: A smartphone seismic network for earthquake early warning and beyond. Science advances, 2(2), e1501055.

Kong, Q., Allen, R. M., \& Schreier, L. (2016b). MyShake: Initial observations from a global smartphone seismic network. Geophysical Research Letters, 43(18), 9588-9594.

Lee, S., Suh, J., Choi, Y. (2018). Review of smartphone applications for geoscience: current status, limitations, and future perspectives, Earth Sci. Informatics.

Lima, W.S., Souto, E., El-Khatib. K., vd. (2019). Human activity recognition using inertial sensors in a smartphone: An overview, Sensors (Switzerland). doi: 10.3390/s19143213.

Lipton, Z.C., Berkowitz, J., Elkan, C. (2015). A critical review of recurrent neural networks for sequence learning, arXiv preprint arXiv: 1506.00019.

Liu, J., Chen, R., Chen, Y., vd. (2012a). iParking: An intelligent indoor location-based smartphone parking service, Sensors (Switzerland). doi: 10.3390/s121114612.

Liu, J., Chen, R., Pei, L., vd. (2012b). A hybrid smartphone indoor positioning solution for mobile LBS, Sensors (Switzerland). doi: $10.3390 / \mathrm{s} 121217208$.

Majumder, S., Deen, M.J. (2019). Smartphone sensors for health monitoring and diagnosis, Sensors (Switzerland).

Manos, A., Klein, I., Hazan, T. (2019). Gravity-based methods for heading computation in pedestrian dead reckoning, Sensors (Switzerland). doi: 10.3390/s19051170.

Real Ehrlich, C., Blankenbach, J. (2019). Indoor localization for pedestrians with real-time capability using multi-sensor smartphones, Geo-Spatial Inf Sci. doi: 10.1080/10095020.2019.1613778

Retscher, G. (2019). Indoor Altitude Determination Using MEMS-based Sensors in Smartphones, In Proceedings of the ION 2019 Pacific PNT Meeting (pp. 615-627).

Sherstinsky, A. (2020). Fundamentals of recurrent neural network (RNN) and long short-term memory (LSTM) network. Physica D: Nonlinear Phenomena, 404, 132306.

Voicu, R.A., Dobre, C., Bajenaru, L., Ciobanu RI (2019) Human physical activity recognition using smartphone sensors. Sensors (Switzerland). doi: 10.3390/s19030458

Zhuang, Y., Yang, J., Li, Y., vd. (2016). Smartphone-based indoor localization with bluetooth low energy beacons, Sensors (Switzerland). doi: 10.3390/s16050596. 